\title{
On the use of discrimination to assess memory
}

\author{
BERNT CHRISTIAN SKOTTUN \\ Skottun Research, Piedmont, Califormia
}

\begin{abstract}
A number of studies have made use of sensory discrimination to assess memory. These experiments have typically employed a temporal two-alternative forced choice paradigm in which the interstimulus interval (ISI) is varied. Many of these tests have shown that ISI has little or no effect on discrimination performance. This has been taken as evidence for perfect or very good memory. An alternative interpretation would be that discrimination tests lack sufficient sensitivity to reveal memory effects. In the present study, computer simulations based on signal detection theory were used to estimate the relative effects of memory noise and direct noise (i.e., noise inherent in the discrimination task) on discrimination performance. These simulations indicate that the amount of memory noise has to be quite large to cause a marked elevation in discrimination thresholds and that sensory discrimination is a relatively insensitive test of memory.
\end{abstract}

In a number of studies, visual discrimination has been used to assess the accuracy of memory. In these tests a subject is asked to compare two stimuli that are separated by some amount of time. The general result of this research has been that the amount of time separating these two stimuli has little or no effect on the discrimination threshold (Bennett \& Cortese, 1996; Blake, Cepeda, \& Hiris, 1997; Greenlee, Rischewski, Mergner, \& Seeger, 1993; Lee \& Harris, 1996; Magnussen \& Dyrnes, 1994; Magnussen \& Greenlee, 1992; Magnussen, Greenlee, Asplund, \& Dyrnes, 1990, 1991; Magnussen, Greenlee, \& Thomas, 1996; Magnussen, Idås, \& Myhre, 1998; Magnussen, Landrø, \& Johnsen, 1985; Nilson \& Nelson, 1981; Regan, 1985; Vogels \& Orban, 1986). These findings have been taken as evidence for very good or even "perfect" memory (Magnussen, 2000; Magnussen \& Greenlee, 1999; Reinvang, Magnussen, Greenlee, \& Larsson, 1998). This interpretation carries with it two assumptions: (1) that memory has a limiting influence on discrimination performance and (2) that the memory happens to be so accurate that it does not degrade the performance. An alternative interpretation would be that discrimination performance is too insensitive a test to detect inaccuracies in memory.

In order to be able to interpret a null result obtained with a test designed to measure some underlying phenomenon one needs to know the sensitivity or resolution of the test, relative to changes in the underlying phenomenon. Without this knowledge one cannot decide whether the absence of a measured effect really reflects no change in the underlying phenomenon or simply re-

Correspondence concerning this article should be addressed to B. C. Skottun, Skottun Research, P.O. Box 592, Ramah, NM 87321.

Note-This article was accepted by the previous editorial team, headed by Neil Macmillan. flects the limited resolution of the test. In the case of the use of sensory discrimination to assess memory, the sensitivity of the test itself, relative to the accuracy of the memory, has yet to be determined. Given the large number of studies that have shown little or no effect of memory on discrimination performance, it seemed reasonable to attempt to gain some insight into this issue.

Although it is generally assumed that any inaccuracy in memory would cause discrimination thresholds to increase, it is not clear how much a given amount of inaccuracy would be expected to elevate the thresholds. This leaves open the possibility that a substantial amount of inaccuracy in memory would have only a modest effect on discrimination performance. In this case, what appears as good memory might simply mean that memory has little impact on discrimination performance - that is, that discrimination is an insensitive test of memory. In the present study, computer simulations were used to investigate this possibility.

Discrimination tests in which the stimuli to be discriminated are presented simultaneously result in finite discrimination thresholds; thus, there clearly are factors that limit discrimination itself. When a subject is required to compare two stimuli presented at different times, memory becomes a factor that has to be added to the factors that determine threshold when the stimuli are presented simultaneously. It is generally assumed that discrimination performance with simultaneously presented stimuli is determined by noise in the representations of the stimuli (Bradley, Skottun, Ohzawa, Sclar, \& Freeman, 1987; Green \& Swets, 1966). The role of memory in this case would be to contribute additional noise to the noise inherent in the representations. This means that discrimination performance would be determined by some combination of memory noise and direct noise, where direct noise refers to the noise involved in generating representations directly - that is, without memory-from the sensory system. The relative contribution of noise from 
these two sources is not known and may vary from one kind of discrimination task to another.

When memory noise is added to a discrimination task, the effect on performance will be determined by three factors: (1) the amount of direct noise, (2) the amount of memory noise, and (3) the mechanism whereby the two types of noise are combined. In the present work the combining of the noise was carried out by adding random variables (see the Method section). Depending on the nature of this mechanism, it may be possible for a substantial amount of memory noise to have only a minor degrading effect on discrimination performance. In this case, the observed lack of an effect of interstimulus interval (ISI) on discrimination performance may reflect no or very little memory involvement in the discrimination performance, rather than perfect or very good memory. As will be seen below, this not only is a possibility but may, in fact, be exactly what one ought to expect.

\section{METHOD}

In the present study, the noise of the direct representations of the two stimuli to be discriminated, as well as the noise introduced by memory, was modeled by Gaussian distributions. The memory noise was added to one of the direct distributions by the following formula: $s_{\mathrm{d}+\mathrm{m}}=\sqrt{ }\left(s_{\mathrm{d}}{ }^{2}+s_{\mathrm{m}}{ }^{2}\right)$, where $s_{\mathrm{d}}, s_{\mathrm{m}}$, and $s_{\mathrm{d}+\mathrm{m}}$ represent the standard deviations of the distributions of the direct noise, of the memory noise, and of the combined noise. If we express the memory noise as a fraction $k$ of the direct noise, so that $s_{\mathrm{m}}=k s_{\mathrm{d}}$, we get: $s_{\mathrm{d}+\mathrm{m}}=s_{\mathrm{d}} \sqrt{\left(1+k^{2}\right)}$.

Discrimination performance was assessed using receiver operating characteristic (ROC) analysis (see Bradley et al., 1987, for details) and was carried out for pairs of distributions. In each pair, one distribution contained the combination of direct noise and memory noise, and the other distribution consisted of direct noise only. ROC analyses were carried out for 151 different separations of the means (varied in a steps of 1/50 the standard deviation of the direct distribution alone) for each of 11 different amounts of memory noise (varied in steps of $0.2 s_{\mathrm{d}}$ ).

\section{RESULTS}

When the task is to discriminate between two stimuli presented at the same time (e.g., side by side), most people would assume that discrimination is limited by the noise in the representations of the two stimuli-that is, by direct noise alone. If one were to change the experimental paradigm so that one stimulus was presented before the other (i.e., so that the first stimulus is terminated before the second appears), one would then have to assume that discrimination was performed by comparing the representation of the second stimulus (generated directly from sensory stimulation) with the representation of the first stimulus, which was now mediated by memory. In this case, the representation of the first stimulus would incorporate a combination of direct noise and memory noise. The addition of memory noise would be expected to cause the discrimination threshold to increase. Conversely, any lack of increase in threshold would, according to this reasoning, be taken to mean that memory contributed no noise - that is, that memory was perfect or, at the very least, very good.

Figure 1 shows the standard deviation of the combined distribution (expressed as a fraction of the standard deviation of the direct noise alone) as a function of the standard deviation of the memory noise (also expressed as a fraction of the standard deviation of the direct distribution). Generally, Figure 1 shows that adding memory noise to direct noise causes only a relatively modest increase in the standard deviation of the combined distribution.

To assess the effect of memory noise on the discrimination threshold, theoretical values of the percentage of correct responses in a two-alternative forced choice (2AFC) paradigm were determined from the area under the ROC curve (see Bradley et al., 1987) when the standard deviation was $s_{\mathrm{d}+\mathrm{m}}$ in the representation of one stimulus and

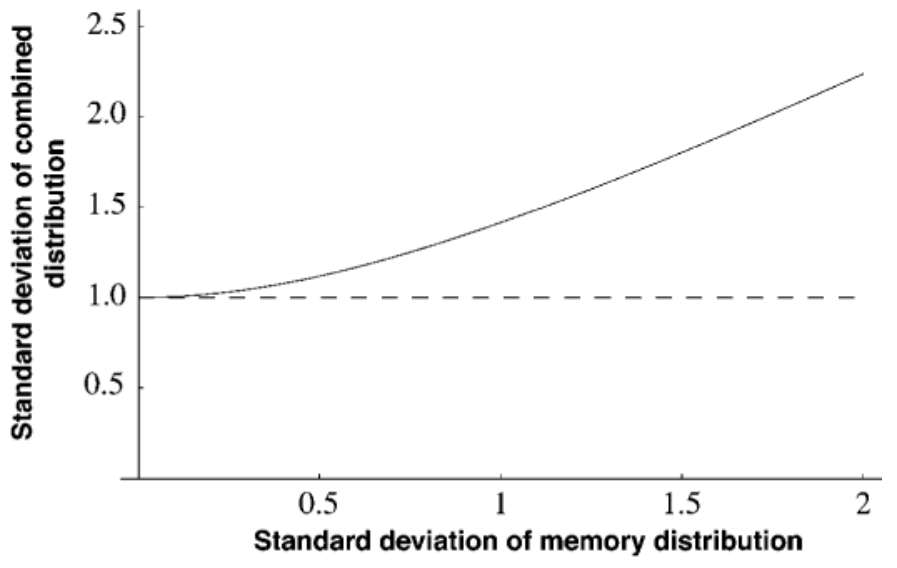

Figure 1. The standard deviation of the combination of direct noise and memory noise as a function of the standard deviation of the memory noise. The standard deviation of the direct noise (dashed line) was held constant at 1.0. 
$s_{\mathrm{d}}$ in that of the other. This kind of ROC analysis has the advantage that it allows one to estimate 2AFC discrimination performance without having to make assumptions about the underlying distributions. Figure $2 \mathrm{~A}$ plots percentage of correct responses as a function of the separation between the means of the two distributions. The parameter is the standard deviation of the memory noise $\left(s_{\mathrm{m}}=0\right.$ to 2 in 0.2 steps). Figure $2 \mathrm{~A}$ shows that the function relating percentage correct to the degree of separation between the means of the two distributions becomes shallower as $s_{\mathrm{m}}$ increases.

Conventionally, in a discrimination experiment, the amount of separation required to achieve $75 \%$ correct discrimination is defined as the discrimination threshold. The discrimination threshold (i.e., the separation required for $75 \%$ correct performance) is shown as a function of the magnitude of the memory noise in Figure 2B. In this plot two things should be noted. (1) The curve is quite flat. This means that an increase in memory noise tends to cause only a modest increase in discrimination threshold. This is particularly pronounced when the memory noise is small. (2) The overall increase in threshold resulting from adding memory noise is, in general, relatively modest. Only when the noise introduced by memory is very large is there a pronounced increase in threshold. For instance, in order to double the discrimination threshold (relative to the one in the no-memory condition), the standard deviation of the memory noise has to be 2.4 times that of the direct noise.

\section{DISCUSSION}

The examples above have indicated that it may be possible for memory to introduce a considerable amount of noise into a discrimination task without this resulting in a pronounced increase in the discrimination threshold. For instance, adding an amount of memory noise equal to the direct noise results in only a $22 \%$ increase in threshold (Figure 2B). In the case of spatial frequency discrimination, the threshold is typically on the order of $5 \%$
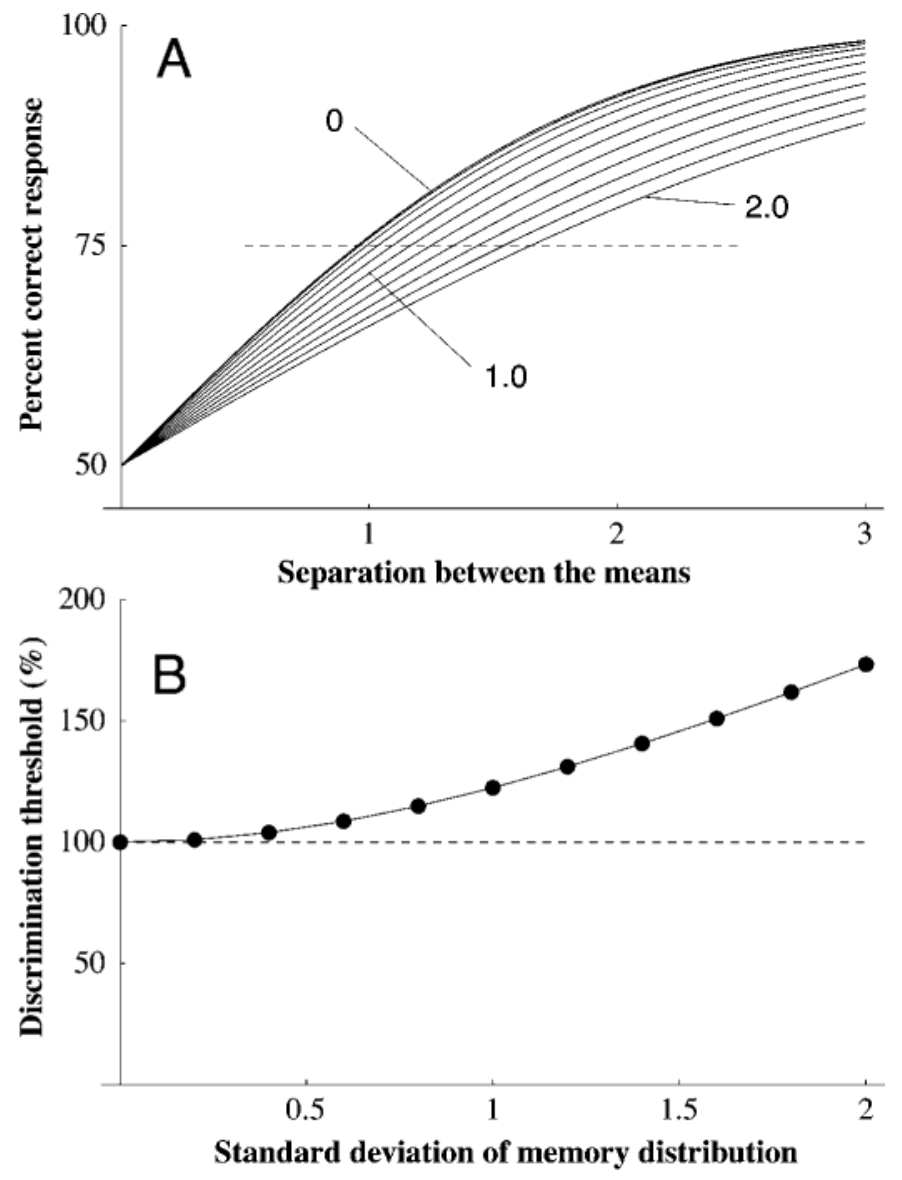

Figure 2. (A) Theoretical values of percentage correct in a twoalternative forced choice experiment (computed from the area under the receiver operating characteristic curve; see the text) as a function of the separation between the means of the two distributions. The parameter is the standard deviation of the memory noise. (B) The $75 \%$ discrimination threshold in $\mathrm{A}$ as a function of the standard deviation of the memory noise. 
(approximately 0.07 octaves; Campbell, Nachmias, \& Jukes, 1970). A $22 \%$ increase of a $5 \%$ threshold (i.e., $22 \%$ of $5 \%$ ) yields a discrimination threshold of $6.1 \%$. This would be considered a very small increase and could easily be overlooked if there were variability (e.g., intersubject variability) in the data. In this case, it may be concluded that memory is very good or even perfect. Yet, as we saw, there is a considerable amount of memory noise present. In other words, memory is not perfect.

In the case of orientation discrimination, the threshold of human observers is typically about $0.5^{\circ}$ (Andrews, 1967; Bradley \& Skottun, 1984). Adding 22\% to this value gives us a threshold of about $0.6^{\circ}$. An increase from $0.5^{\circ}$ to $0.6^{\circ}$ is likely to be overlooked or dismissed. Again, the likely conclusion will be that the memory is perfect or, at least, very good. As was the case for spatial frequency, this would be a misleading, if not a directly erroneous conclusion, given that a considerable amount of memory noise is present. These considerations show that it is entirely possible for memory to be "involved" in a discrimination task and for there to be a considerable amount of memory noise without this resulting in a pronounced elevation of the threshold. In fact, it may be possible for there to be a considerable amount of memory noise without there being any detectable increase in discrimination threshold. Judging from the above analyses, this is not only possible, but likely.

Although it would seem that memory noise equal in magnitude to direct noise would mean that memory is poor, some might argue that memory noise of this magnitude is small and that this is a case of good memory. If so, there would be two points to be made. (1) If the meaning of good memory and poor memory cannot be agreed upon, this would mean that a quantitative approach (such as the present one) is needed. (2) The present analysis was stimulated by some rather bold claims that memory was "perfect" in some discrimination tasks. Clearly, if memory noise is equal to direct noise, memory is not perfect.

There are two reasons why memory noise has little effect on discrimination performance: (1) the process of adding two random variables makes memory noise have relatively little effect on the combined distribution, and (2) memory noise affects only one of the two direct distributions (i.e., when a comparison is made across time between two stimuli, memory affects only the representation of the first of them). These considerations assume no stimulus-response nonlinearities. Also, the computations are based on the notion of an ideal observer (i.e., a decision maker who makes optimal use of all available information), which carries with it the assumption that there is no noise in the decision process itself. Compressive nonlinearities and decision noise could reduce the impact of memory on the discrimination performance even further.

In conclusion, the present analyses indicate that discrimination tasks may be rather insensitive tests of memory and that a lack of increase in discrimination thresholds when large ISIs are introduced does not preclude the presence of a considerable amount of memory noise. Therefore, it seems that it may be difficult to draw firm conclusions regarding the accuracy of memory from discrimination experiments.

\section{REFERENCES}

Andrews, D. P. (1967). Perception of contour orientation in the central fovea: Part I. Short lines. Vision Research, 7, 975-997.

Bennett, P. J., \& Cortese, F. (1996). Masking of spatial frequency in visual memory depends on distal, not retinal, frequency. Vision Research, 36, 233-238.

BLAKE, R., CEPEDA, N. J., \& HiRIS, E. (1997). Memory for visual motion. Journal of Experimental Psychology: Human Perception \& Performance, 23, 353-369.

BRADLEY, A., \& SKOTTUN, B. C. (1984). The effects of large orientation and spatial frequency differences on spatial discriminations. Vision Research, 24, 1889-1896.

Bradley, A., Skottun, B. C., Ohzawa, I., Sclar, G., \& Freeman, R. D. (1987). Visual orientation and spatial frequency discrimination: A comparison of single neurons and behavior. Journal of Neurophysiology, 57, 755-772.

Campbell, F. W., Nachmias, J., \& Jukes, J. (1970). Spatial frequency discrimination in human vision. Journal of the Optical Society of America, 60, 555-559.

GREEN, D. M., \& SwETS, J. A. (1966). Signal detection theory and psychophysics. New York: Wiley.

Greenlee, M. W., Rischewski, J., Mergner, T., \& Seeger, W. (1993). Delayed pattern discrimination in patients with unilateral temporal lobe damage. Journal of Neuroscience, 13, 2565-2574.

LEE, B., \& HARRIS, L. (1996). Contrast transfer characteristics of visual short-term memory. Vision Research, 36, 2159-2166.

Magnussen, S. (2000). Low-level memory processes in vision. Trends in Neurosciences, 23, 247-251.

MAGNuSSEN, S., \& DYRNES, S. (1994). High-fidelity perceptual longterm memory. Psychological Science, 5, 99-102.

Magnussen, S., \& GReEnleE, M. W. (1992). Retention and disruption of motion information in visual short-term memory. Journal of Experimental Psychology: Learning, Memory, \& Cognition, 18, 151156.

Magnussen, S., \& Greenlee, M. W. (1999). The psychophysics of perceptual memory. Psychological Research, 62, 81-92.

Magnussen, S., Greenlee, M. W., Asplund, R., \& Dyrnes, S. (1990). Perfect visual short-term memory for periodic patterns. European Journal of Cognitive Psychology, 2, 345-362.

Magnussen, S., Greenlee, M. W., Assplund, R., \& Dyrnes, S. (1991). Stimulus-specific mechanisms of visual short-term memory. Vision Research, 31, 1213-1219.

Magnussen, S., Greenlee, M. W., \& Thomas, J. P. (1996). Parallel processing in visual short-term memory. Journal of Experimental Psychology: Human Perception \& Performance, 22, 202-212.

MAGNuSSEN, S., IDÅs, E., \& MyHRE, S. H. (1998). Representation of orientation and spatial frequency in perception and memory: A choice reaction-time analysis. Journal of Experimental Psychology: Human Perception \& Performance, 24, 707-718.

MAGNUSSEN, S., LANDRø, N. L., \& JoHNSEN, T. (1985). Visual halffield symmetry in orientation perception. Perception, 14, 265-273.

Nilson, T. H., \& Nelson, T. M. (1981). Delayed monochromatic hue matches indicate characteristics of visual memory. Journal of Experimental Psychology: Human Perception \& Performance, 7, 141-150.

REGAN, D. (1985). Storage of spatial-frequency information and spatialfrequency discrimination. Journal of the Optical Society of America $A, 2,619-621$.

Reinvang, I., Magnussen, S., Greenlee, M. W., \& Larsson, P. G. (1998). Electrophysiological localization of brain regions involved in perceptual memory. Experimental Brain Research, 123, 481-484.

Vogels, R., \& Orban, G. A. (1986). Decision processes in visual discrimination of line orientation. Journal of Experimental Psychology: Human Perception \& Performance, 12, 115-132.

(Manuscript received October 1, 2002; revision accepted for publication March 4, 2004.) 